\title{
NUMERICAL MODELING OF CONCENTRATION POLARIZATION IN REVERSE OSMOSIS DESALINATION SYSTEMS
}

\author{
Ali K. Abdel-Rahman \\ Associate Professor, Department of Mechanical Engineering, \\ Faculty of Engineering, Assiut University, Assiut 71516, EGYPT \\ e-mail: nada ali54@yahoo.com
}

(Received December 26, 2007 Accepted January 13, 2008)

Fresh water is rapidly becoming a scarce resource in many countries around the world. Modern desalination technologies, applied to seawater and brackish water, offer effective alternatives in a variety of circumstances. Out of the several desalination processes considered, reverse osmosis is one of the major processes used in desalination. Reverse osmosis is in general the most economical process for desalination of brackish water and seawater. As widely accepted technology, reverse osmosis became more and more competitive and is superior to the traditional thermal process when a comparison is made of capital investment and energy consumption.

The present study pertains to modeling numerically of a seawater desalination system. The proposed method considers solving the momentum, energy and mass transfer conservation equations for a smallscale reverse osmosis system. The solution of the conservation equations was obtained using the SIMPLE (Semi-Implicit Method for Pressure Linked Equations) pressure-correction scheme and the high order discretization scheme QUICK (Quadratic Upwind Interpolation Convective Kinematics) is employed for the discretization of convection terms in the frame of staggered grid. The model was verified using the experimental data from the literature. Parameter sensitivity was carried out to select the proper time and spatial step sizes. The calculations were run for a very long time enabling a prediction of operational performance at high overall system recoveries. Effects of the feed temperature variations on the operating parameters are also considered in this study.

KEYWORDS: Crossflow membrane, Fouling, Reverse osmosis, Numerical modeling, Mass transfer, Concentration polarization.

\section{INTRODUCTION}

Fresh water is rapidly becoming a scarce resource in many countries around the world. In Egypt, the conventional water resources from the River Nile and the available wells are barely able to cover the increasing demand in the valley and in the Red Sea areas. The increased standard of living and the expansion of petroleum industries and tourism have led to the gap between supply and demand [1]. Khalil et al. [2] outlined the 
different methods of seawater desalination appropriate for the Egyptian environment and recommended the critical path for the selection of the technology to be used in each particular site.

\section{NOMENCLATURE}

$\begin{array}{ll}\text { Alphabetic Symbols } \\ c & \text { concentration }(\mathrm{kg} / \mathrm{kg}) \\ c_{w} & \text { concentration near membrane } \\ & \text { wall }(\mathrm{kg} / \mathrm{kg}) \\ c_{b} & \text { feed concentration }(\mathrm{kg} / \mathrm{kg}) \\ c_{p} & \text { specific heat capacity }(\mathrm{kJ} / \mathrm{kg} \mathrm{K}) \\ D & \text { Diffusion coefficient }\left(\mathrm{m}^{2} / \mathrm{s}\right) \\ f & \text { rejection of the membrane } \\ h & \text { channel half-width }(\mathrm{m}) \\ k_{t} & \text { thermal conductivity }(\mathrm{W} / \mathrm{m} \mathrm{K}) \\ L & \text { channel length }(\mathrm{m}) \\ P & \text { pressure }(\mathrm{Pa}) \\ R e_{o} & \text { inlet Reynolds number }=2 h u_{m o} / v \\ S_{\Phi} & \text { source term of the variable } \phi \\ S_{p} & \text { coefficient in the discretized } \\ & \text { source term } \\ S_{u} & \text { coefficient in the discretized } \\ & \text { source term } \\ t & \text { time }(\mathrm{s}) \\ T & \text { temperature }\left({ }^{\circ} \mathrm{C}\right)\end{array}$

\begin{tabular}{|c|c|}
\hline$u$ & mean streamwise velocity $(\mathrm{m} / \mathrm{s})$ \\
\hline$u_{m o}$ & $\begin{array}{l}\text { inlet cross-sectionally averaged } \\
\text { streamwise velocity }(\mathrm{m} / \mathrm{s})\end{array}$ \\
\hline$v$ & velocity in y direction $(\mathrm{m} / \mathrm{s})$ \\
\hline$v_{w}$ & wall velocity $(\mathrm{m} / \mathrm{s})$ \\
\hline$v_{w o}$ & $\begin{array}{l}\text { wall velocity at the beginning of } \\
\text { permeation }(\mathrm{m} / \mathrm{s})\end{array}$ \\
\hline$x$ & $\begin{array}{l}\text { Cartesian coordinate in the } \\
\text { streamwise direction }\end{array}$ \\
\hline$y$ & $\begin{array}{l}\text { Cartesian coordinate normal to } \\
\text { the wall }\end{array}$ \\
\hline \multicolumn{2}{|c|}{ Greek Symbols } \\
\hline$\delta$ & $\begin{array}{l}\text { thickness of the concentration } \\
\text { polarization layer }(\mathrm{m})\end{array}$ \\
\hline$\phi$ & any of variables to be solved \\
\hline$\Gamma_{\Phi}$ & $\begin{array}{l}\text { diffusion coefficient of the } \\
\text { variable } \phi\end{array}$ \\
\hline$\mu$ & dynamic viscosity (Pa .s) \\
\hline 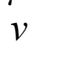 & kinematics viscosity $=\mu / \rho$ \\
\hline$\rho$ & density $\left(\mathrm{kg} / \mathrm{m}^{3}\right)$ \\
\hline
\end{tabular}

Large-scale thermal desalination technologies have been in use since the 1950 s. The larger desalination plants have provided fresh water supplies for drinking, municipal use and agricultural development, particularly in the Gulf States. In the past, high capital costs and heavy energy consumption generally translated into excessive desalinated water costs. However, advances in technology have helped to drastically reduce capital and running costs as well as energy requirements, rendering desalination more viable an option than ever before [3].

New high-performance processes, predominantly based on high-performance membrane technologies such as nanofiltration (NF) and reverse osmosis (RO), were developed and first applied by the industry in the 1970s and 1980s, with the promise of further cost reductions and process simplification [3]. Reverse osmosis is the most popular technology for seawater desalination. During the last two decades hundreds of reverse osmosis seawater desalination plants have been built worldwide. Each year the plant sizes and cost-effectiveness have increased. Recently the reverse osmosis has achieved growing acceptance as an economical and viable alternative to multi-stage flash distillation (MSF) process for desalting seawater [4, 5].

Nevertheless, the RO process is severely affected by transient build up of a concentration polarization $(\mathrm{CP})$ layer of rejected species at the membrane upstream 
interface [6-8]. Particle accumulation in the CP layer provides an additional resistance to permeate flow and, hence, reduces permeate flux [9]. Prediction of concentration profiles of particles at the concentration polarization layer and its effect on permeate flux and solute rejection is a necessary step in the design of an efficient system in the RO processes [8]. Different theoretical models such as the film model, the gel polarization model, the osmotic pressure model, and the resistance in series model have been widely used to predict concentration polarization and its effects on permeate flux and solute rejection [7].

A number of investigators have carried out the work on different aspects of reverse osmosis seawater desalination. A1-Bastaki and Abbas [10] have used a simple model to predict the performance of hollow fiber RO membranes. Later, they studied the effect of ignoring concentration polarization and pressure drops on the recovery and the salt rejection [11]. They found that ignoring concentration polarization and pressure drops results in significant overestimation of the recovery.

Moreover, RO system performances are in general limited by several factors, e.g. the feed pressure, the feed salinity (TDS or total dissolved solids), the permeate flux (proportional to the desired desalting capacity), etc. . . . In addition to specific membrane performance characteristics, each of these parameters is affected, under different conditions, by the feed temperature [12]. Peter et al. [13] have considered the pretreatment of the feed with ultrafiltration (UF) membranes since the RO desalination plants depend on high quality feed water to ensure reliable and stable operation of the RO system. They concluded that UF pretreatment also allows the economical utilization of RO membranes in areas where membrane desalination has not been considered as the appropriate technology due to difficult raw water conditions.

Computational fluid dynamics (CFD), together with mass transfer modeling, have been proved to be a powerful tool to be used in the feed-side of membrane modules to facilitate the predictions of velocities, pressure and solute concentration, variables that are crucial for the management of the concentration polarization phenomenon $[14,15]$. One or more of the following simplifications had been made in the previous studies: (a) the wall permeation velocity was assumed piece-wise or constant along the axial length; (b) the fluid flow field was approximated by some prescribed functions or by a reduced form of the momentum equation; (c) the wall velocity may depend on osmotic pressure but axial pressure drop was neglected or an approximate pressure drop was used without solving the momentum equation; and (d) the fluid transport properties were assumed constant or concentration-dependent viscosity and diffusion coefficient were used. Limited number of the previous studies has realized the sensitivity of the feed solution properties to the changes in both temperature and concentration.

The present study pertains to modeling numerically of a seawater desalination system. The study is directed towards removing some of the assumptions discussed above and to establish a numerical method capable of predicting the flow and concentration characteristics of the RO desalination system taking into account the variable properties of the stream. For this purpose, a finite volume discrete scheme using the SIMPLE (Semi-Implicit Method for Pressure Linked Equations) pressurecorrection scheme combined with QUICK (Quadratic Upwind Interpolation Convective Kinematics) scheme in the frame of staggered grid are used. 


\section{MODEL PROBLEM AND DISCRETIZATION}

\subsection{Governing Equations}

Two types of membrane modules, spiral wound and hollow-fiber configurations, dominate the desalination and water treatment systems. In the unwound spiral wound membrane the feed stream, which flows mainly tangentially to the porous membrane surface, is modeled by the two dimensional Navier-Stokes, convective and diffusion mass and energy transfer equations.

For two dimensional, incompressible, unsteady laminar channel flows; the continuity and Navier-Stokes equations are given as [16];

$$
\begin{aligned}
& \frac{\partial \rho}{\partial t}+\frac{\partial u}{\partial x}+\frac{\partial v}{\partial y}=0 \\
& \frac{\partial(\rho u)}{\partial t}+\frac{\partial(\rho u u)}{\partial x}+\frac{\partial(\rho v u)}{\partial y}-\frac{\partial}{\partial x}\left[\mu\left(\frac{\partial u}{\partial x}\right)\right]-\frac{\partial}{\partial y}\left[\mu\left(\frac{\partial u}{\partial y}\right)\right]+\frac{\partial P}{\partial x}=0 \\
& \frac{\partial(\rho v)}{\partial t}+\frac{\partial(\rho u v)}{\partial x}+\frac{\partial(\rho v v)}{\partial y}-\frac{\partial}{\partial x}\left[\mu\left(\frac{\partial v}{\partial x}\right)\right]-\frac{\partial}{\partial y}\left[\mu\left(\frac{\partial v}{\partial y}\right)\right]+\frac{\partial P}{\partial y}=0
\end{aligned}
$$

Where, $u$ and $v$ are the streamwise and transverse velocity components, respectively, $P$ is the pressure, $\rho$ is the density and $\mu$ is the dynamic viscosity.

Mass transfer occurring within domains with porous walls can be mathematically expressed by the two dimensional convective and diffusion equation as follows [16];

$$
\frac{\partial(\rho c)}{\partial t}+\frac{\partial(\rho u c)}{\partial x}+\frac{\partial(\rho v c)}{\partial y}-\frac{\partial}{\partial x}\left[D\left(\frac{\partial c}{\partial x}\right)\right]-\frac{\partial}{\partial y}\left[D\left(\frac{\partial c}{\partial y}\right)\right]=0
$$

Where $D$ is the solute diffusion coefficient and $c$ is the solute concentration.

Most of the previous models solve equations (1)-(4) using constant or concentration-dependent only thermophysical and flow properties. In the present study, the energy equation has to be solved to account for the temperature dependence of the abovementioned properties. For two dimensional, incompressible, unsteady laminar channel flows; the energy equation is given as [16]

$$
\frac{\partial(\rho T)}{\partial t}+\frac{\partial(\rho u T)}{\partial x}+\frac{\partial(\rho v T)}{\partial y}-\frac{\partial}{\partial x}\left[\frac{k_{t}}{c_{p}}\left(\frac{\partial T}{\partial x}\right)\right]-\frac{\partial}{\partial y}\left[\frac{k_{t}}{c_{p}}\left(\frac{\partial T}{\partial y}\right)\right]=0
$$

where $k_{t}$ and $c_{p}$ are the fluid thermal conductivity and specific heat at constant pressure, respectively.

\subsection{Computational Domain and Grid System}

The physical problem considered in the present study is unsteady state laminar channel flow with permeable walls. Figure 1 displays the flow geometry and the coordinate system used for the problem considered. The origin of the streamwise coordinate, $x$, is 
located at the beginning of the membrane and the origin of the normal coordinate, $y$, is set at the horizontal midplane of the channel. The position of the inlet section for the computational domain is located at a position $L_{\text {Imp }}$ upstream from the leading edge of the membrane, and that of the outlet section is located at a position $L_{P e r m}$ downstream from the leading edge of the membrane. The main result were obtained for a computational domain of $-10 \leq x / h \leq 100$, i.e. $L_{I m p}=10 \mathrm{~h}$ and $L_{P e r m}=100 \mathrm{~h}$.

One of the important points of numerical solution is the choice of grid point system for which the equations are discretized. In relation with the use of the SIMPLE scheme, a staggered grid is used in this work [17]. A nonuniform grid system was employed and the grid density was made higher near the leading edge of the membrane in $x$ direction and near the walls in the $y$ direction to take into accounts the steep change of velocity, temperature and concentration there. Grid points of total number of 56x 50 were allocated in the computational domain. Numerical experiments using different sets of grids were carried out in order to prove the sufficiency of the selected grid system.

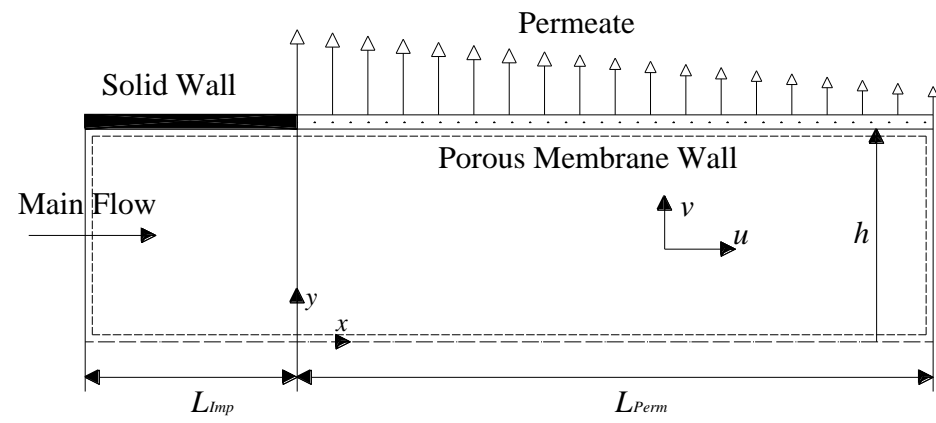

Figure 1 Schematic diagram of flow geometry and computational domain.

\subsection{Discretization}

The unsteady-state form of the conservation equations of continuity, momentum, concentration and energy can be written in a general form as $[14,17]$;

$$
\frac{\partial(\rho \phi)}{\partial t}+\frac{\partial(\rho u \phi)}{\partial x}+\frac{\partial(\rho v \phi)}{\partial y}-\frac{\partial}{\partial x}\left[\Gamma_{\phi}\left(\frac{\partial \phi}{\partial x}\right)\right]-\frac{\partial}{\partial y}\left[\Gamma_{\phi}\left(\frac{\partial \phi}{\partial y}\right)\right]-S_{\phi}=0
$$

where $\phi$ stands for any of the variables to be solved, $\Gamma_{\Phi}$ is the diffusion coefficient, and $S_{\Phi}$ is the source term of the variable $\phi$. For $\phi=u$ or $v$ and $\Gamma_{\Phi}=\mu$ one gets the momentum equations, while for $\phi=1$ and $\Gamma_{\Phi}=0$ one obtains the continuity equation [18]. If $\phi=T$ and $\Gamma_{\Phi}=k_{t} / c_{p}$ one gets the energy equation. When $\phi=c$ and $\Gamma_{\Phi}=D$, the general equation stands for the mass transfer equation $[14,19]$.

All the governing equations are discretized by first integrating them over a control volume $(\mathrm{CV})$ and then approximating the fluxes of variable crossing every faces of each cell in term of the values at the neighboring grid points. In the present work, a QUICK scheme, which can handle uniform and non-uniform grid systems, is used to finite difference the convective terms and to secure second order accuracy in central differencing the diffusive fluxes. The resulting finite-difference equations are described in the form of [20] 


$$
\begin{array}{ll}
a_{p} \phi_{p}=\sum_{i} a_{i} \phi_{i}+S_{u} \Delta V+a_{p}^{o} \phi_{p}^{o}, & i=E, W, N, S, E E, W W, N N, S S, \text { (7a) } \\
a_{p}=\sum_{i} a_{i}+a_{p}^{o}-S_{p} \Delta V, & i=E, W, N, S, E E, W W, N N, S S,(7 \mathrm{~b})
\end{array}
$$

where $\Delta V$ is the cell volume and $S_{p}$ and $S_{u}$ are the coefficient appearing in the following linearized source term;

$$
S_{\phi}=S_{u}+S_{p} \phi
$$

The finite difference coefficients $a_{i}$ are the coefficients describing the magnitudes of the sum of the convective and diffusive fluxes and contain the geometric properties of the control volume. They are given in [20].

\subsection{Boundary Conditions}

The boundary conditions of the problem are specified as the followings:

1- At the inlet, the flow is assumed to be fully developed thus a parabolic flow is specified. A uniform inflow concentration of $c_{o}$ is specified. A constant inlet temperature $T_{o}$ is specified.

2- At the symmetry plane, the transverse velocity $v=0$, and the normal gradients of the tangential velocity $u$, the concentration and temperature are set to zero.

3- At the membrane walls, the conditions are more complex, as flow permeates through the wall. For a membrane located at the plane $y=h$, a constant rejection and variable permeation rate were specified. The tangential velocity $u$ is set to zero i.e. no slip at membrane wall. Temperature at the membrane wall $\mathrm{T}_{\mathrm{w}}$ was set to a constant value. Variation in permeation was modeled using the following expression based on the osmotic model [8];

$$
v_{w}(x, t)=A[\Delta P(x, t)-\Delta \pi(x, t)],
$$

where $A$ is the membrane permeability, and $\Delta P$ and $\Delta \pi$ are the applied and the induced osmotic back pressure. The osmotic pressure is described as a linear function of the solute concentration as [21]

$$
\pi=K_{\text {osm }} c
$$

where $K_{o s m}$ is the osmotic pressure coefficient.

The boundary condition of the concentration at the membrane walls results from a balance of the convective and diffusive fluxes. In addition, it must be taken into account the fact that not all of the solute permeates through the membrane. This is done via the use of a rejection coefficient, $f$, and the concentration boundary condition is given by;

$$
D \frac{\partial c}{\partial y}+v_{w} f c_{w}=0
$$

4- At the exit, both the flow, temperature and concentration fields are assumed to obey the boundary layer approximation. It is important to mention that this treatment of the down stream end boundary condition has proved to be robust 
and effective in shortening the computational domain leading to the reduction of the number of grid nodes [18, 20].

\section{$2.5 \quad$ Numerical procedure}

The present study utilizes a modified version of the SIMPLE procedures developed by Patankar and Spalding [17]. The main steps of the SIMPLE algorithm are;

1- A pressure field is assumed,

2- It is used to obtain approximate velocity field,

3- The velocity and pressure fields are corrected if the former does not satisfy the continuity equation,

4- Solve the discretization equations for the other $\phi$ 's such as temperature and concentration providing their influence on the flow field.

5- Return to step 2 with the corrected velocity field and the new values of all other $\phi$ 's and then the process 2-4 are repeated until a converged solution is obtained.

In the present work, the cross-stream distribution of $u$-velocity component is adjusted to satisfy the overall continuity (conservation of the mass flow are integrated over a cross-stream line) whereas the pressure field is adjusted to satisfy the overall momentum balance. This procedure is important especially for the present problem in which the flow is changed as the flow moves downstream due to the suction of the permeate flux from the membrane surface [18, 20]. Moreover, the cross-stream distribution of the concentration is adjusted to satisfy the overall mass concentration of the permeated species.

An alternating direction implicit (ADI) procedure, which has been developed by Abdel-Rahman and Suzuki [18], has been combined with the iterative solution procedure of equations (7) to enhance isotropic propagation of a change of variables occurring at one point to the surrounding. This procedure makes use of the line-by-line Tri-Diagonal Matrix Algorithm (TDMA) solver. In the ADI procedure, sweep of lineby-line integration was carried out along both north-south grid lines and along eastwest grid lines alternatively. The same procedure was applied twice for the pressure correction [20].

\subsection{Physical Properties}

The membrane solute rejection leads to the development of a solute concentration profile in the fluid phase adjacent to the membrane. Moreover, the feed is subjected to temperature variations in order to study its effects on the performance of the reverse osmosis desalination system. Therefore, the transport and physical properties of the solutions in the transport equations (1)-(5), should include the variation with the solute concentration and the solution temperature. The correlations relative to the variation with the concentration and temperature of the physical and transport properties of the studied aqueous solution of sodium chloride are collected from different sources [19, 22-28].

For validation of the present model, the physical properties of the solution were taken as the same of those used by Geraldes et al. [19] which vary with the 
solution concentration only. However, in the rest of this study the fluid used was an aqueous solution of sodium chloride of varying physical and transport properties with temperature and concentration. Expressions for the variation of physical properties (densities and enthalpies) with temperature and concentration for sodium chloride aqueous solutions were taken from Sparrow [22]. Sparrow [22] presented equations for the thermodynamic properties of aqueous solution of sodium chloride as function in temperature and concentration. These equations are valid for temperatures from 0 to $300{ }^{\circ} \mathrm{C}$, and concentrations extending to saturation with suitable accuracy. Sparrow's equations for density and enthalpy have the following forms

$$
\begin{aligned}
& \rho=a_{0}+a_{1} T+a_{2} T^{2}+a_{3} T^{3}+a_{4} T^{4} \\
& h=b_{0}+b_{1} T+b_{2} T^{2}+b_{3} T^{3}+b_{4} T^{4}
\end{aligned}
$$

where $\rho$ and $h$ are the solution density $\left(\mathrm{kg} / \mathrm{m}^{3}\right)$ and enthalpy (kJ/kg), respectively. $a_{i}{ }^{\prime} \mathrm{s}$ and $b_{i}$ 's are coefficients function in solution concentration (c) [22]. $T$ is the solution temperature in ${ }^{\circ} \mathrm{C}$. It is worth mentioning that the results obtained for solution density using Sparrow's equation (10a) compared very well with that obtained by Geraldes et $a l$. [19] for sodium chloride solution at $25^{\circ} \mathrm{C}$ as shown in Fig. 2.

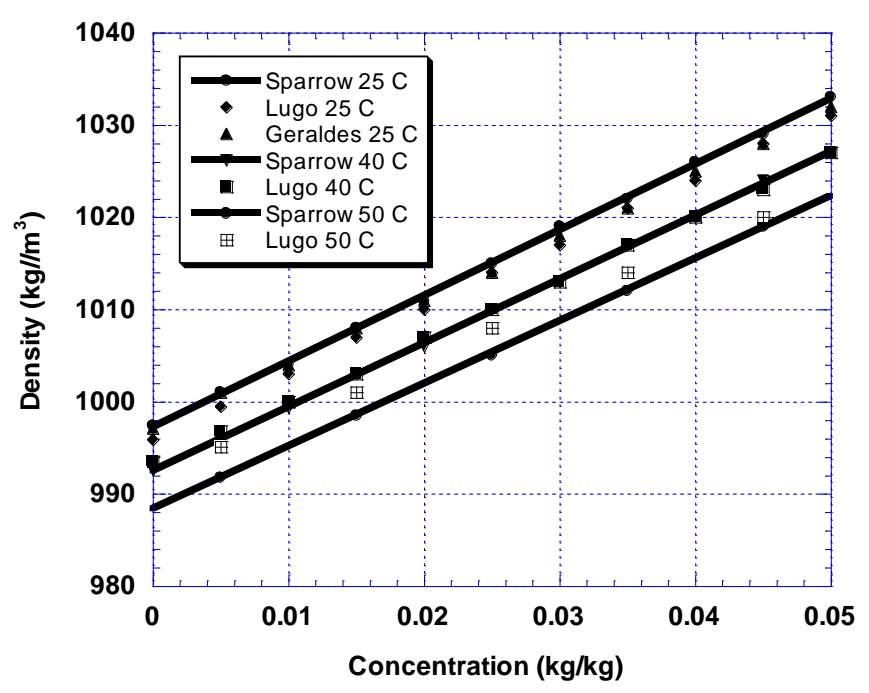

Figure 2 Density of sodium chloride solution as function of concentration and temperature.

Lugo et al. [23] have presented an easy-to-use accurate method to calculate some of the thermophysical properties of aqueous solutions by excess functions approach. The excess function method is used for the calculation of thermophysical properties by evaluating the discrepancies between ideal and real states. Lugo et al. [23] generalized the procedure of calculating any thermophysical property $\Pi(c, T)$ by the following equation

$$
\Pi(c, T)=\left[\left(1-\frac{c}{c_{e u}}\right) \Pi^{w}+\frac{c}{c_{e u}} \Pi^{e u}+E_{c}\right]\left(1+A_{c} T\right)
$$


where $T$ is expressed in ${ }^{\circ} \mathrm{C}$ and the superscript $w$ and $e u$ (eutectic) stands for "water" and the solution at $c=c_{e u}, E_{c}$ is the excess function and $A_{c}$ is a composition-dependent coefficient. In the present study, equation (10c) is used to calculate heat capacities, and thermal conductivities of sodium chloride aqueous solution as function in temperature and concentration. Figure 2 shows also that the density results obtained using Lugo et al. [23] correlation equation (10c) compared very well with that obtained by Sparrow [22] up to $40{ }^{\circ} \mathrm{C}$, and then tend to overestimate it at higher solution temperatures. In the present study, the sodium chloride solution density and enthalpy as function in temperature and concentration are obtained using Sparrow [22] correlation equations $(10 \mathrm{a}, \mathrm{b})$.

Moreira et al. [24] and Chenlo et al. [25] have measured the kinematic viscosities of sodium chloride aqueous solutions at various concentrations and temperatures. The experimental data were correlated with concentration ( $m$ is the molal concentration, mol/ $\mathrm{kg}$ ) and temperature to give the following equation for the kinematic viscosities

$$
\frac{v}{v_{w}}=1+a m \exp \left(\frac{m^{f}}{g T_{R}^{3}+b}\right)
$$

where $T_{R}=T(\mathrm{~K}) / 273.15, a, f, g$, and $b$ are constants of the above correlation equation (with values of $0.014,0.2,-0.127$ and 0.909 respectively), and

$$
v_{w}=0.09607 \times 10^{-6} \exp \left(\frac{2.90}{T_{R}^{3}}\right)
$$

is the water viscosity at the working temperature. Figure 3 shows that the viscosity results obtained using Moreira et al. [24] correlation equation (10d) are in good agreement with that obtained using Geraldes et al. [19] equation for sodium chloride at $25^{\circ} \mathrm{C}$. In the present study, the sodium chloride solution viscosity as function in temperature and concentration is obtained using Moreira et al. [24] correlation equation (10d).

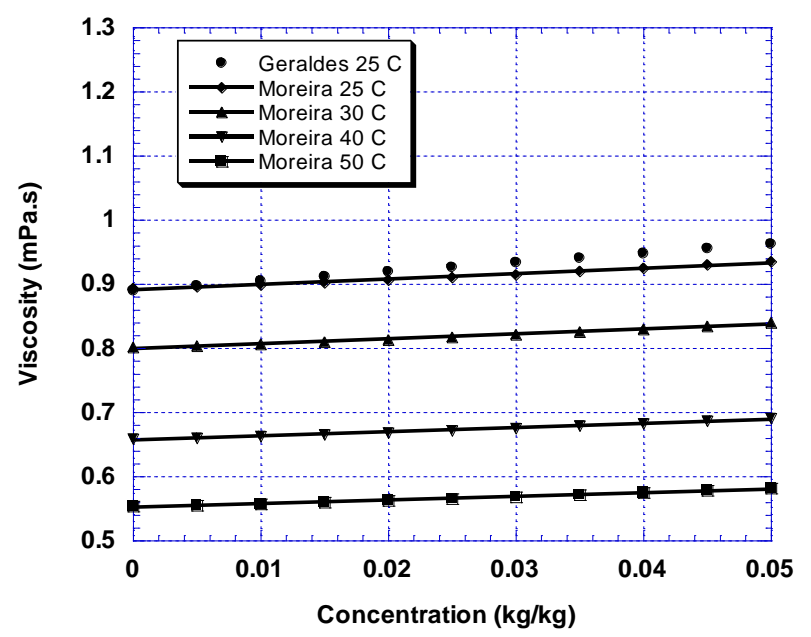

Figure 3 Viscosity of sodium chloride solution as function of concentration and temperature. 
Diffusion in liquids is complicated by nonideal effects, such as the variation in the diffusion coefficient, $\boldsymbol{D}$, with concentration and temperature. Some progress has been made in the theory of diffusion in liquids in that the theory at least predicts what variables to correlate. None of the liquid theories has been developed to the point of engineering usefulness [26].

Brodkey and Hershey [26] reported that Sutherland [27] and Einstein [28] independently derived an equation for the diffusion coefficient by regarding the diffusion flow as a balance between a driving force and a flow resistance. These are usually called hydrodynamic theories. Sutherland [27] and Einstein [28] originally used the gradient of osmotic pressure as the driving force, but more modern developments use chemical potential.

The Stokes-Einstein equation predicts

$D \mu / T=$ constant

where $T$ is the absolute temperature and $\mu$ is the solvent dynamic viscosity. Equation (10e) is reasonably valid for liquids over small changes in viscosity and temperature. Equation (10e) has been widely used as a basis for interpreting experimental data in liquids. In the present study, equation (10e) combined with the correlated equation of Geraldes et al. [19] is used to obtain the diffusion coefficient as function in solution concentration and temperature. Figure 4 shows that the diffusion coefficient obtained in the present study is increased with increasing solution temperature significantly, while the effect of solution concentration variations is insignificant particularly at low temperature.

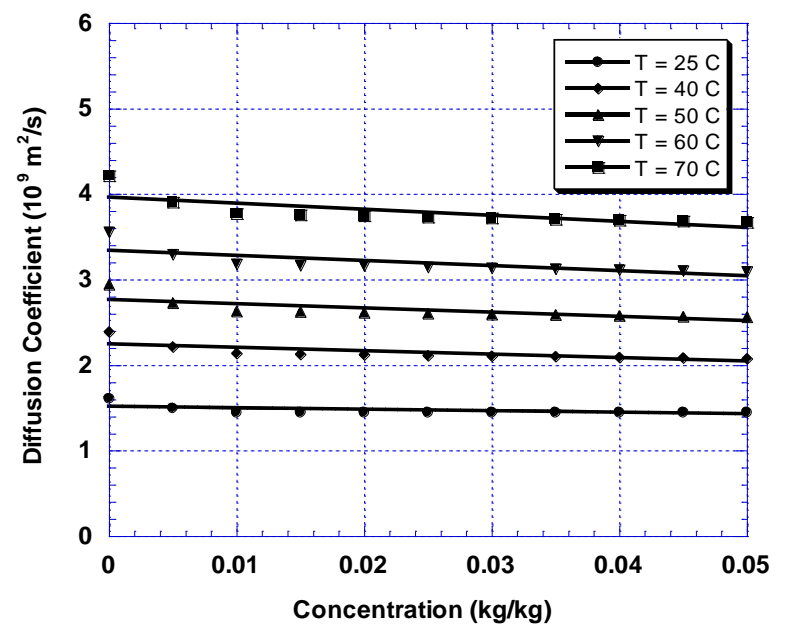

Figure 4 Diffusion coefficient of sodium chloride solution as function of concentration and temperature.

\section{RESULTS AND DISCUSSION}

\subsection{Model Validation}

On the light of the present objective of this work, the validity of the present numerical simulation of the permeate fluxes and concentration polarization in RO processes will 
be verified by comparing results of this present model with the existing experimental data of Kim and Hoek [8] and the analytical film theory. Figure 5 shows the average permeate fluxes predicted by the present model along with the experimental data of Kim and Hoek [8]. The figure shows that the present model is capable of predicting permeate fluxes with a reasonable accuracy. The figure also shows that the present model is capable of predicting the linear relation between the applied pressure and the fluxes (as suggested by equation 8) and that the slope of pressure-flux is slightly smaller than the slope of the experimental data. The numerical predictions obtained by Kim and Hoek [8] shows a similar trend regarding the pressure-flux slope where the predicted slope obtained using different models including their convection-diffusion (CD) model was smaller than the experimental one as shown in Fig. 5.

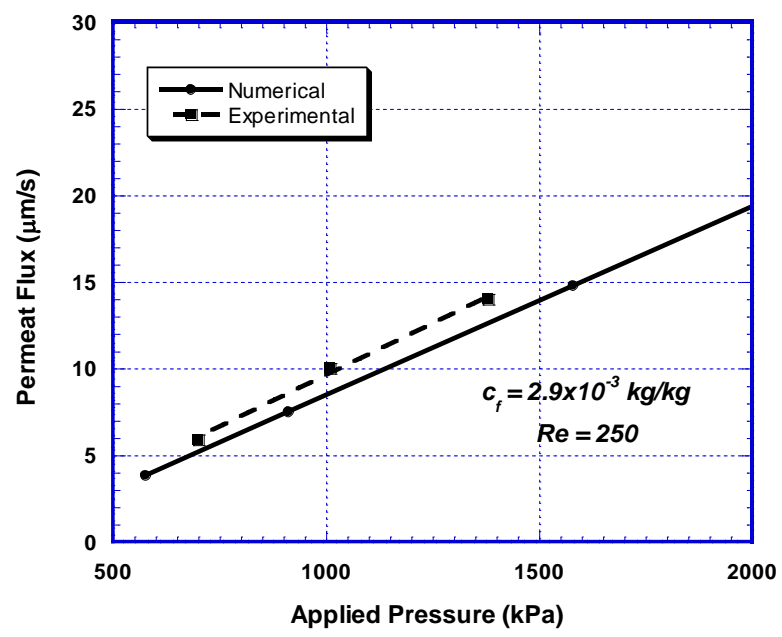

Figure 5 Channel average permeate fluxes predicted by the present model and the experimental data of Kim and Hoek [8].

\subsection{Model Predictions}

In the present study, series of simulations were carried out to examine the predictability power of the present model. Numerical simulations are performed to study the effects of varying the controlling parameters such as the crossflow Reynolds number, applied pressure, salt concentration, and feed temperature on the performance of the RO processes. The crossflow hydrodynamics are varied within the laminar range (Re < 2100), the applied pressures are varied in the range from $500-3000 \mathrm{kPa}$, and salt concentrations are varied from fresh water conditions to about the seawater salinity (e.g. $0.5 \times 10^{-3}-3.0 \times 10^{-3} \mathrm{~kg} / \mathrm{kg}$ ). Moreover, the feed temperatures are varied from $20-$ $45^{\circ} \mathrm{C}$.

Accurate prediction of concentration polarization phenomena is very important for reverse osmosis processes because it enables prediction of the onset of scaling by solute minerals. The film theory (FT) is also used in this study to predict the local concentration polarization modulus, $\mathrm{c}_{\mathrm{w}} / \mathrm{c}_{\mathrm{b}}$ via [8]

$$
\frac{c_{w}(x)}{c_{b}}=\left[1-f+f \exp \left(-\frac{v_{w}(x)}{k(x)}\right)\right]
$$


where $v_{w}(x)$ is the local permeate flux predicted by the present model, $k(x)$ is the local mass transfer coefficient predicted by [8]

$$
k(x)=0.538\left(\frac{\gamma_{w} D^{2}}{x}\right)^{1 / 3}
$$

where $\gamma_{w}=3 u_{m o} / h$ is the wall shear rate [29], $u_{m o}$ is the inlet cross-sectionally averaged streamwise velocity, and $h$ is the channel half height.

Figures 6 and 7 show the local CP modulus, $c_{w}(x) / c_{b}$, predicted by the present model and the FT model for various crossflow Reynolds numbers and applied pressures. Figures 6 and 7 show several facts in RO processes which can be summarized as:

- The wall concentration is increasing as the flow moves downstream due to the accumulation of the solute particles.

- The wall concentration increases rapidly at the starting point of permeation before it reaches a rather constant value before the exit of the channel specially for the higher crossflow Reynolds number and the lower pressure.

- The wall concentration at the same axial location along the channel is increased as the applied pressure is increased and is decreased as the crossflow Reynolds number is increased.

- The predictions made by FT model for the CP modulus overestimated its counterpart values predicted by the present model. This could be accounted for the simplifications imposed on the FT model in comparison with the present model which solve the full momentum and mass transfer equations. This behavior agrees qualitatively with that obtained by Kim and Hoek [8] irrespective of the simplifications used in their model.

- The discrepancies between the predictions of CP modulus made by the present model and FT model become smaller for lower applied pressures and higher crossflow Reynolds numbers.

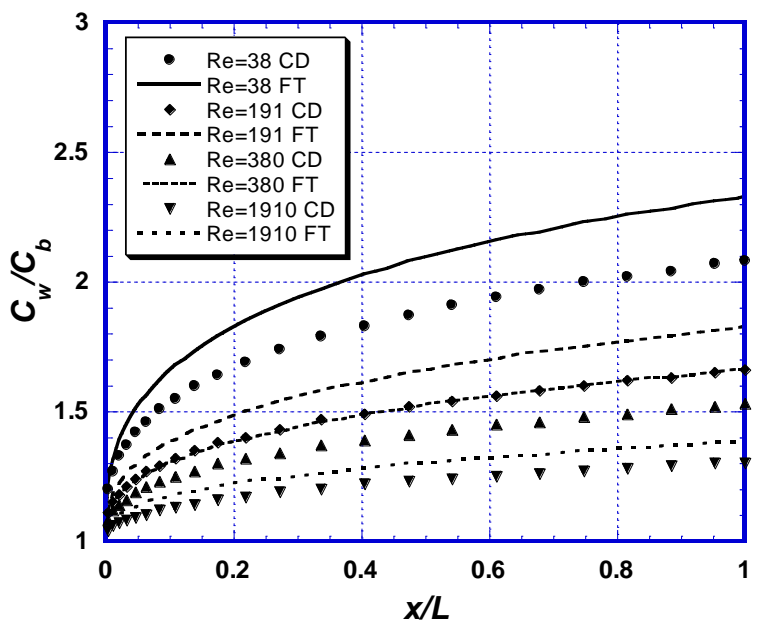

Figure 6 Prediction of local CP modulus depending on different cross flow Reynolds numbers. 


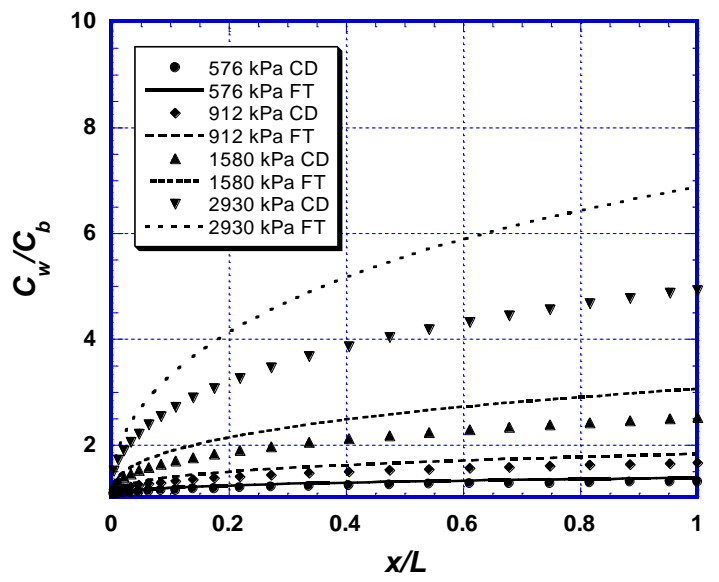

Figure 7 Prediction of local CP modulus depending on different applied pressures.

Effects of variation of both the cross flow Reynolds numbers and the applied pressures on the wall permeation velocity along the membrane channel are shown in Figs. 8 and 9. These numerical computations were carried out to explore the effects of changing the Reynolds numbers and the applied pressures on the permeation fluxes. Figures 8 and 9 show that the permeation velocity decreases as the flow moves downstream along the channel for all cases considered. Figure 8 shows also that the effect of increasing the Reynolds number is to increase the permeation velocity. This can be attributed to the fact that the CP layer thickness is decreased with the increase of the Reynolds number as shown in Fig. 10. Figures 8 and 10 indicate that the permeation velocity increases as the Reynolds number increases at every axial location due to the corresponding decrease of the CP layer thickness with the increase in Reynolds number. The decrease of the CP layer thickness with the increase of Reynolds number is due to the higher shear effect in case of higher Reynolds number which counteract the increase in the convective particles due to the higher permeation.

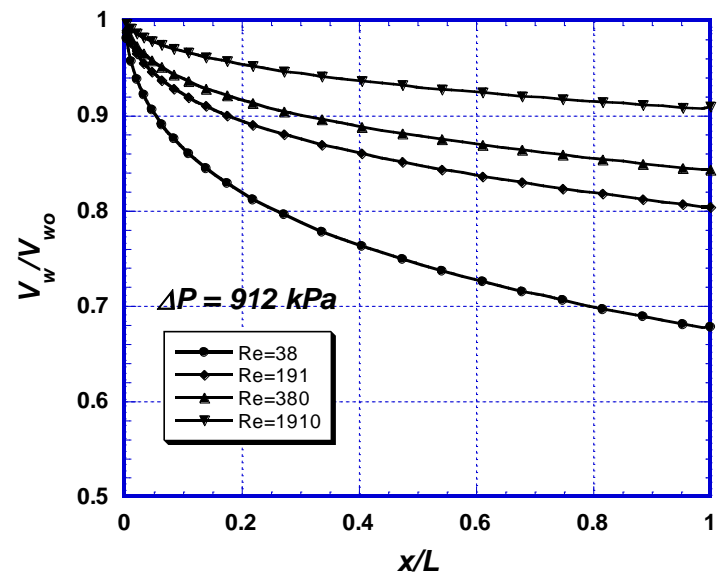

Figure 8 Prediction of local permeation velocity depending on different cross flow Reynolds numbers. 


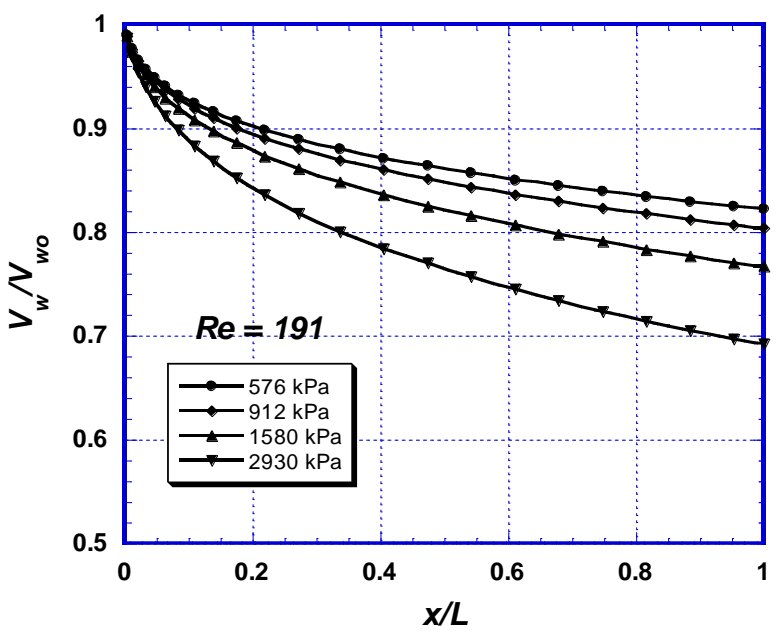

Figure 9 Prediction of local permeation velocity depending on different applied pressures.

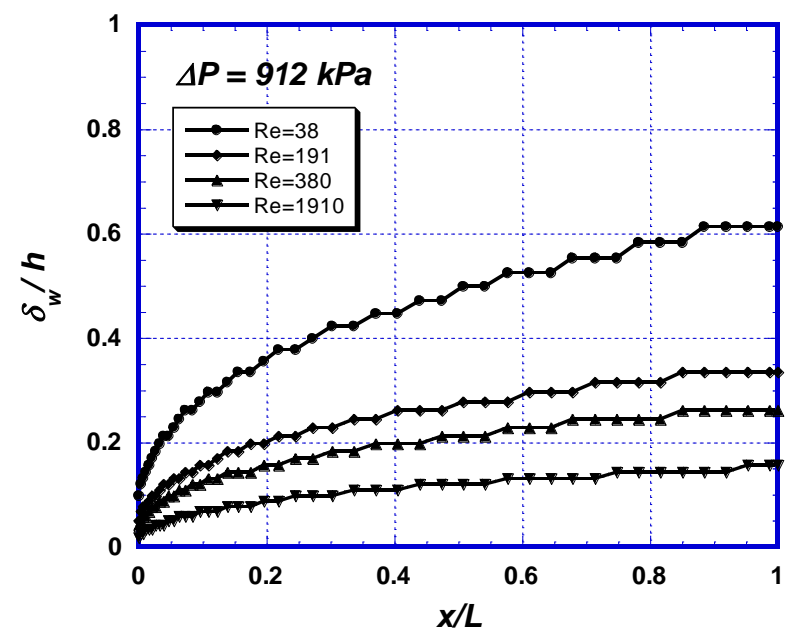

Figure 10 Prediction of local CP thickness depending on different cross flow Reynolds numbers.

The reduction of concentration polarization is important for the improvement of the performance of reverse osmotic type membrane, which will lead to reduction in the fouling of the membrane. Several measures to reduce the concentration polarization in order to control the fouling have been adopted and proposed. One of the techniques used to reduce the concentration polarization is increasing the feed flow rate as shown in Fig. 6. Increasing the feed temperature is another way to increase the productivity of the RO processes. The permeation rate is increased as the feed temperature is increased due to the decrease in the dynamic viscosity. However, the CP thickness is increased as shown in Fig. 11. 


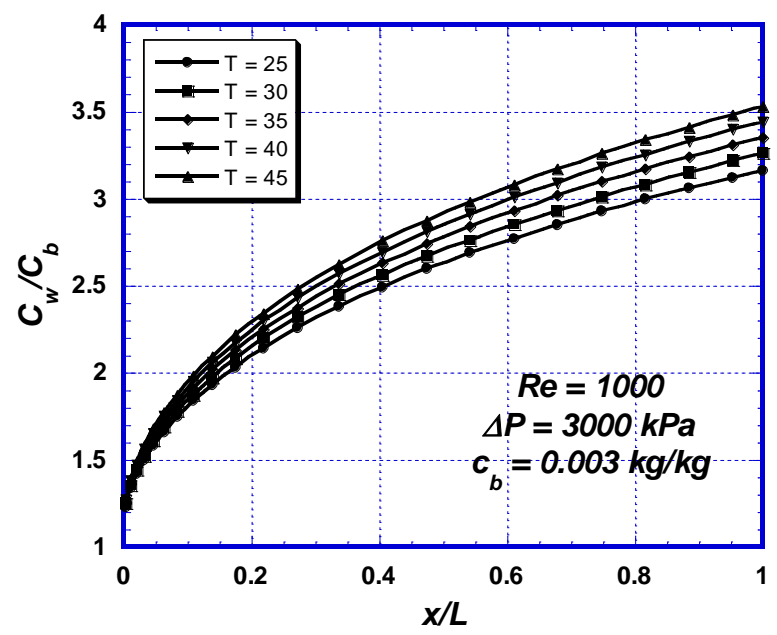

\section{Figure 11 Prediction of local CP thickness depending on different feed temperatures.}

The effects of varying the feed concentrations on the $\mathrm{CP}$ modulus and the local permeation velocity are shown in Figs. 12 and 13. Figure 13 shows that the permeation velocity is higher for the lower feed concentration. This can be attributed to the fact that the osmotic pressure is smaller for the case of lower concentration (refer to equation 8 b). Hence, according to equation (8a), the permeation velocity is higher in the case of lower feed concentration.

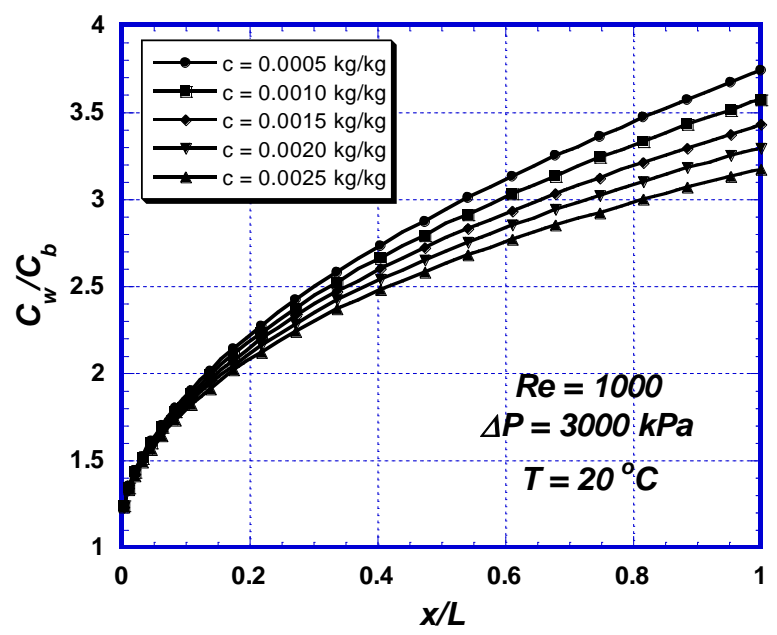

Figure 12 Prediction of local CP thickness depending on different feed concentrations. 


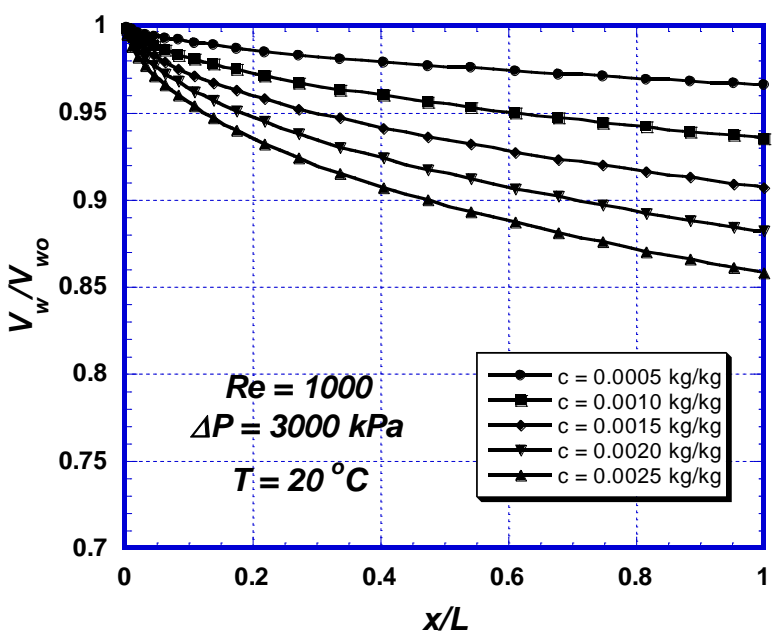

Figure 13 Prediction of local permeation velocity depending on different feed concentrations.

\section{CONCLUSIONS}

RO desalination process has been numerically investigated. This was facilitated by solving the elliptic type of the governing equations using the SIMPLE pressurecorrection algorithm for pressure field in connection with the high order QUICK scheme. The alternating direction implicit ADI scheme, which makes use of the TDMA in solving the resulting coefficient matrix, was used to solve the governing equations to reduce the number of iterations. A scheme to secure the overall mass conservation was also employed. The following points can be drawn from the numerical simulation:

- Present results of numerical simulation of the mass transfer in RO process agree very well with most of the previous studies.

- Present results of numerical simulation can be used to simulate real situation concerning RO processes and its application in seawater and brackish water.

- Present results can be used to examine the performance of the RO desalination systems under different operating conditions.

- Present model can be used as an efficient tool for the accurate predictions of the RO desalination process.

The simulation model developed in this work can be directly applied to a number of industrial RO processes. The code developed here was designed so that any model describing the dependence of permeates flux and solute rejection on process parameters can easily be introduced into the simulation program. The models for modules other than channel could also be handled by this code.

\section{REFERENCES}

[1] Khalil, E. E., Water strategies and technological development in Egyptian coastal areas. Desalination, 165 (2004) 23-30. 
[2] Khlil, E. E., Mariy, A. H. and Marawan, M., Potable water technology transfer and assessment in Egypt. Desalination, 64 (1987) 217-227.

[3] UN ESCWA (Main), Water desalination technologies in the ESCWA member countries. Report No. E/ESCWA/TECH/2001/3, The United Nations, New York, (2001).

[4] Al-Mudaiheem, A. M. and Miyamura, H., Construction and commissioning of Al Jobail Phase II desalination plant. Desalination, 55 (1985) 1-11.

[5] Brandt, D. C., Seawater reverse osmosis: an economic alternative to distillation. Desalination, 52 (1985) 177-186.

[6] Sablani, S. S., Goosen, M. F. A., Al-Belushi, R. and Wilf, M., Concentration polarization in ultrafiltration and reverse osmosis: a critical review. Desalination, 141 (2001) 269-289.

[7] Chen, K. L., Song, L., Ong, S. L. and Ng, W. J., The development of membrane fouling in full-scale RO processes. Journal of Membrane Science, 232 (2004) 63-72.

[8] Kim, S. and Hoek, M. V., Modeling concentration polarization in reverse osmosis processes. Desalination, 186 (2005) 111-128.

[9] Hong, S., Faibish, R. S. and Elimelech, M., Kinetics of permeate flux decline in crossflow membrane filtration of colloidal suspensions. Journal of Colloid and Interface Science, 196 (1997) 267-277.

[10] A1-Bastaki, N. M. and Abbas, A., Modeling an industrial reverse osmosis unit. Desalination, 126 (1999) 33-39.

[11] A1-Bastaki, N. M. and Abbas, A., Predicting the performance of RO membranes. Desalination, 132 (2000) 181-187.

[12] Nisan, S., Commercon, B. and Dardour, S., A new method for the treatment of the reverse osmosis process, with preheating of the feedwater. Desalination, 182 (2005) 483-495.

[13] Peter, H. W., Siverns, S. and Monti, S., UF membranes for RO desalination pretreatment. Desalination, 182 (2005) 293-300.

[14] Geraldes, V., Semião, V. and Pinho, M. N. de, Numerical modeling of mass transfer in slits with semi-permeable membrane walls. Engineering Computations, 17 (2000) 192-217.

[15] Wiley, D. E. and Fletcher, D. F., Techniques for computational fluid dynamics modeling of flow in membrane channels. Journal of Membrane Science, 211 (2003) 127-137.

[16] Anderson, D.A., Tannehill, J.C. and Pletcher, R.H. (1984), Computational fluid mechanics and heat transfer, Hemisphere Publishing Corporation, NewYork.

[17] Patankar, S. V. and Spalding, D. B., A calculation procedure for heat, mass and momentum transfer in three-dimensional parabolic flows. International Journal of Mass and Heat Transfer, 15 (1972) 1787-1806.

[18] Abdel-Rahman, A. K. and Suzuki, K. (1995), Laminar channel flow with fluid injection accounting for the flow in the porous wall, Proceedings of the 5th Int. Conference of Fluid Mechanics, Cairo, pp. 367-379.

[19] Geraldes, V., Semião, V. and Pinho, M.N.de, Flow and mass transfer modelling of nanofiltration. Journal of Membrane Science, 191(2001) 109-128. 
[20] Abdel-Rahman, A. K. (1992), Flow and heat transfer characteristics of internal flows with fluid injection, $\mathrm{PhD}$ thesis, Kyoto University, Kyoto, Japan.

[21] Madiredddi, K., Babcock, R. B., Levine, B., Kim, J. H. and Stenstrom, M. K., An unsteady-state model to predict concentration polarization in commercial spiral wound membranes. Journal of Membrane Science, 157 (1999) 13-34.

[22] Sparrow, B. S., Empirical equation for the thermodynamic properties of aqueous sodium chloride. Desalination, 159 (2003) 161-170.

[23] Lugo, R., Fournaison, L., Chourot, J.-M. and Guilpart, J., An excess function method to model the thermophysical properties of one-phase secondary refrigerants, 25 (2002) 916-9233.

[24] Moreira, R., Chenlo, F. and Pereira, G., Viscosities of ternary aqueous solutions with glucose and sodium chloride employed in osmotic dehydration operation. Journal of Food Engineering, 57 (2003) 173-177.

[25] Chenlo, F., Moreira, R., Pereira, G. and Ampudia A., Viscosities of aqueous solutions of sucrose and sodium chloride of interest in osmotic dehydration process. Journal of Food Engineering, 54 (2002) 347-352.

[26] Brodky, R. S. and Hershey, H. C. (1988), Transport phenomena: A modified approach, McGraw Hill International edition, New York.

[27] Sutherland, W., Philos. Mag., 9, 781 (1905).

[28] Einstein, A., Ann. Physik, 4, 17, 549 (1905).

[29] Davis, R. H., Modeling of fouling of cross-flow microfiltration membranes, Separation and Purification Methods, 21 (1992) 75.

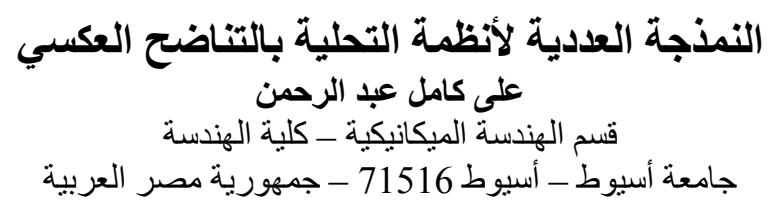

أصبحت المياه العذبة من الموارد النادرة بصورة سريعة في العديد من البلدان حول العالم. توفر تقنيات

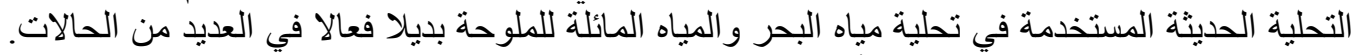

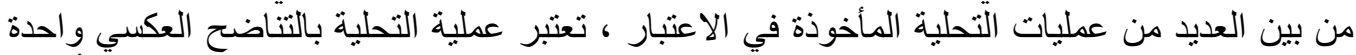

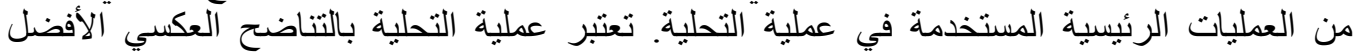

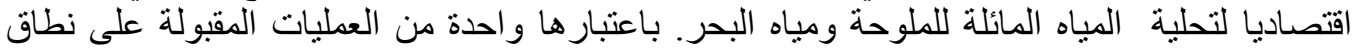

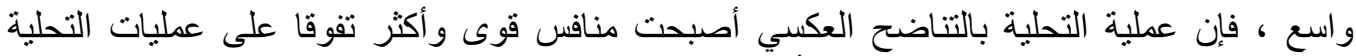

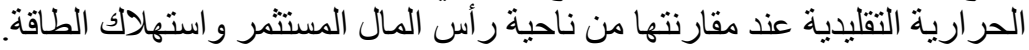

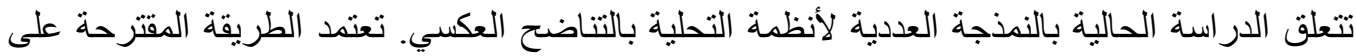

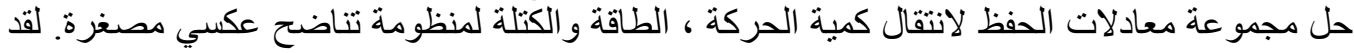

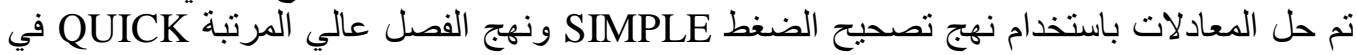

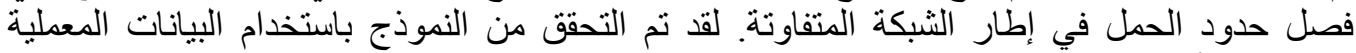

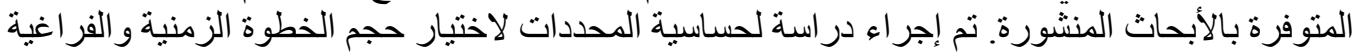

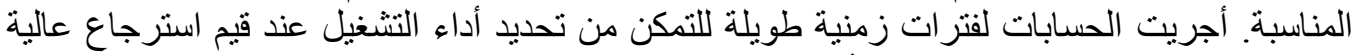
للنظام. كما تم في هذه الدر اسة اعنبار تأثير التغير طريل في درجة دن حرارة التغذية على محددات التشغيل. 